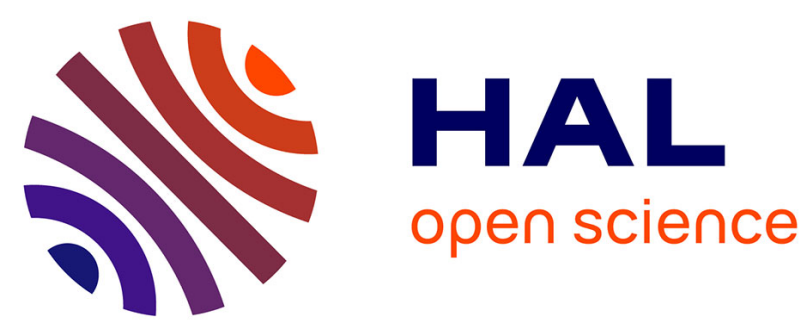

\title{
Optimal pump excitation frequency for improvement of damage detection by nonlinear vibro acoustic modulation method in a multiple scattering sample
}

Nesrine Houhat, Vincent Tournat, Sébastien Ménigot, Tarek Boutkedjirt, Jean Marc Girault

\section{To cite this version:}

Nesrine Houhat, Vincent Tournat, Sébastien Ménigot, Tarek Boutkedjirt, Jean Marc Girault. Optimal pump excitation frequency for improvement of damage detection by nonlinear vibro acoustic modulation method in a multiple scattering sample. Applied Acoustics, 2019, 155, pp.222-231. 10.1016/j.apacoust.2019.06.010 . hal-02155298

\section{HAL Id: hal-02155298 \\ https://hal.science/hal-02155298}

Submitted on 13 Jun 2019

HAL is a multi-disciplinary open access archive for the deposit and dissemination of scientific research documents, whether they are published or not. The documents may come from teaching and research institutions in France or abroad, or from public or private research centers.
L'archive ouverte pluridisciplinaire HAL, est destinée au dépôt et à la diffusion de documents scientifiques de niveau recherche, publiés ou non, émanant des établissements d'enseignement et de recherche français ou étrangers, des laboratoires publics ou privés. 


\title{
Optimal pump excitation frequency for improvement of damage detection by nonlinear vibro acoustic modulation method in a multiple scattering sample
}

\author{
Nesrine Houhat* \\ Research Center in Industrial Technologies CRTI, P. O. Box 64, Chéraga 16014, Algiers, \\ Algeria, \\ Faculty of Physics, University of Sciences and Technology Houari Boumediene, USTHB, \\ BP 32, El-Alia, DZ-16111 Algiers, Algeria, \\ n.houhat@crti.dz \\ Vincent Tournat \\ LAUM, CNRS UMR 6613, Le Mans Université, Le Mans, France \\ vincent.tournat@univ-lemans.fr \\ Sébastien Ménigot \\ ESEO Group, Angers, France, \\ LAUM, CNRS UMR 6613, Le Mans Université, Le Mans, France, \\ sebastien.menigot@eseo.fr \\ Tarek Boutkedjirt \\ Faculty of Physics, University of Sciences and Technology Houari Boumediene, USTHB, \\ BP 32, El-Alia, DZ-16111 Algiers, Algeria, \\ tboutekdjirt@usthb.dz \\ Jean- Marc Girault \\ ESEO Group, Angers, France, \\ LAUM, CNRS UMR 6613, Le Mans Université, Le Mans, France, \\ jean-marc.girault@eseo.fr
}

Abstract

${ }^{*}$ Corresponding author.

Email address: houhat.nesrine@yahoo.fr (Nesrine Houhat) 
We present a method to systematically optimize nonlinear damage detection in multiple scattering media by the nonlinear Vibro-Acoustic Modulation (VAM) technique. The latter consists here of exciting a medium simultaneously with a high frequency ultrasonic sinusoidal burst and with a low frequency continuous sinusoidal wave. Modulation of the high frequency (probe) by the low frequency (pump) is made possible by the presence of nonlinear scatterers, i.e. cracks, defects. A signal processing technique consisting of a closed loop system drives the automatic adaptation of the pumping frequency, yielding to the optimization of the nonlinear modulation (NM) of the output probing coda signal without a priori information on the medium and the scatterers. The correlation coefficient between a reference output probe signal without the pumping wave and an output modulated probe signal with a pumping wave was considered as our cost function. A multiple scattering solid beam where nonlinear scatterers can be controllably added or removed is designed and tested. The first step of this study is an empirical search of the correlation coefficient dependency on the pumping frequency to verify the performances of the proposed method. Then the implemented optimization algorithm based on genetic algorithm (GA) is used to find automatically the optimal pumping frequency. The obtained optimization results show a good agreement with the empirical study. Moreover, the genetic algorithm allowed to find the optimal pump frequency adapted to each configuration of nonlinear scatterers. This relatively fast search of the optimal nonlinear response could be extended to nonlinear scatterer imaging applications using the information on the resonant modes spatial shapes together with the associated optimal response. 
Keywords: Optimal command, Genetic algorithm, Nonlinear VibroAcoustic Modulation, Crack detection.

1

2

\section{Introduction}

Nonlinear ultrasonic nondestructive testing has known a great development during the last decades. Numerous studies have shown the possibility to detect damages at earlier stages than whith linear ultrasonic methods. The integrity of the structure can be easily preserved by an earlier maintenance. The presence of a nonlinear defect, such as a crack or a delamination, can give rise to nonlinear phenomena, among which are the generation of higher harmonics, subharmonics, wave modulation or resonance frequency shift. Several methods based on the detection of these phenomena have been proposed $[1,2,3,4,5,6,7]$. One of the most widely studied methods is the Vibro-Acoustic Modulation (VAM) [8, 9] or the Nonlinear Wave Modulation Spectroscopy (NWMS) [10], which belongs to the class of nonlinear modulation or nonlinear mixing methods. With the VAM, micro-damage can be detected by following the amplitude modulation induced on a probe signal (e.g. high frequency ultrasound) by a pump signal (e.g. low frequency vibration). Nonlinear modulation techniques have been widely used on samples with simple geometries in which coherent waves propagate $[11,12]$. However, the use of these methods has been poorly studied in environments with complex geometries, which lead to multiple scattering of the waves. Among the few reported results in such case, Zhang et al. have demonstrated that a global inspection of multiple scattering materials is possible by combining the Coda Wave Interferometry (CWI) technique with a nonlinear modulation 
method [13, 14].

Generally, the pump frequency is chosen to correspond to one of the vibration modes of the studied sample in order to amplify the vibration response $[9,15,16,17,18]$. A preliminary modal analysis is, then, necessary for identifying and selecting the resonance frequencies of the sample. It is important to underline that such a procedure requires a further experimental setup and time. Furthermore, a possible drawback of a modal excitation is that the crack or the damaged zone can be located at a strain node of the pump or probe wave, which compromises the generation of the nonlinear modulation. To overcome this problem, a frequency swept pump and/or probe signals have been used $[13,17,19,20,21,18,22]$. As such, several resonance modes are excited and an averaged effect over a wide frequency range is detected, without low sensitivity zones. Dunn et al. suggest that selecting the resonant frequencies as a pumping frequency is not ideal, because the system nonlinearities are maximum at those frequencies and the resonant peaks shift in frequency with changing amplitude [23].

In order to optimize and to refine the sensitivity of the nonlinear modulation method to detect damage, various signal processing techniques have been adopted. These methods make it possible to extract the nonlinear modulation information in the frequency domain [20, 21, 24, 25, 26, 27, 28]. The VAM was also successfully associated with the time reversal technique for localizing and imaging damage in materials [29, 30, 31]. All these postprocessing techniques aim for improving the crack detection with fixed excitations, while it has been proved that the effect of excitation parameters, such as the frequency, can improve the VAM sensitivity [18, 22]. Pieczonka et 
al. have used a swept sine chirp probe excitation and modal frequency pump excitation associated with an advanced signal proccesing technique to find the optimal probe frequency enhancing the VAM sensitivity [17]. Recently, a novel method in which the pump frequency is omitted and the high frequency is amplitude- modulated and contains 3 frequencies that interact in the presence of nonlinearity has been reported [32]. However, to our knowledge, no study using VAM has tried to find the optimal excitation that improve the sensitivity of the damage detection automatically by a closed loop system.

Previous results using the optimal command principle for nonlinear systems were initiated by Ménigot et al. [33, 34] in medical ultrasound imaging. A first attempt in NDT was applied in [35] then in [36]. The originality of these results is to search for the optimal input excitation parameter/shape without any a priori knowledge on the studied system, by using known optimization algorithms. The key point of this method lies in the choice of the cost function which must best write the optimization purpose.

The present study constitutes an extension to the NDT domain, especially, to the VAM technique, of the method demonstrated in [33], in which the optimal command was applied to medical ultrasonic imaging. A conventional VAM system is, then, replaced by a closed loop VAM system permitting a real time optimization of input excitations parameters. We focused, here, on the pump frequency parameter since the nonlinear modulation intensity increase with the pump amplitude $[18,37,38]$. The aim of our study is therefore to find automatically the best pumping frequency which maximizes the nonlinear modulation occurring between the pump and the probe signals without a priori information. The proposed method requires no preliminary 
modal analysis and use a simple optimization genetic algorithm. In a first time, the cost function must be adapted to the VAM. An empirical search of the cost function behavior as a function of the optimization parameter was achieved in order to show the relevance of our choices.

The remainder of the paper is organized as follow, in the Section 2, we present our closed loop optimization system for the VAM technique. Section 3 describes the experiment including the studied sample and the setup. The Section 4 is devoted to the experimental results of the empirical optimization, a modal analysis for a comparison purpose, and the genetic algorithm optimization. Finally, a discussion and conclusion are given in Section 5.

\section{Closed Loop Pump Frequency Optimization}

4 i chosen. Here, only an iterative optimization procedure is implemented. The conventional open loop system is replaced by a closed loop system in which the transmitted pump frequency is modified by adding a feedback, ensuring the optimization of the cost function. The closed loop system of the VAM optimization is described in Fig. 1.

\subsection{Cost function}

As mentioned above, the goal of our study is to find automatically the best pump frequency $f_{p}^{*}$, which maximizes the Nonlinear modulation (NM) 


\section{Open loop}

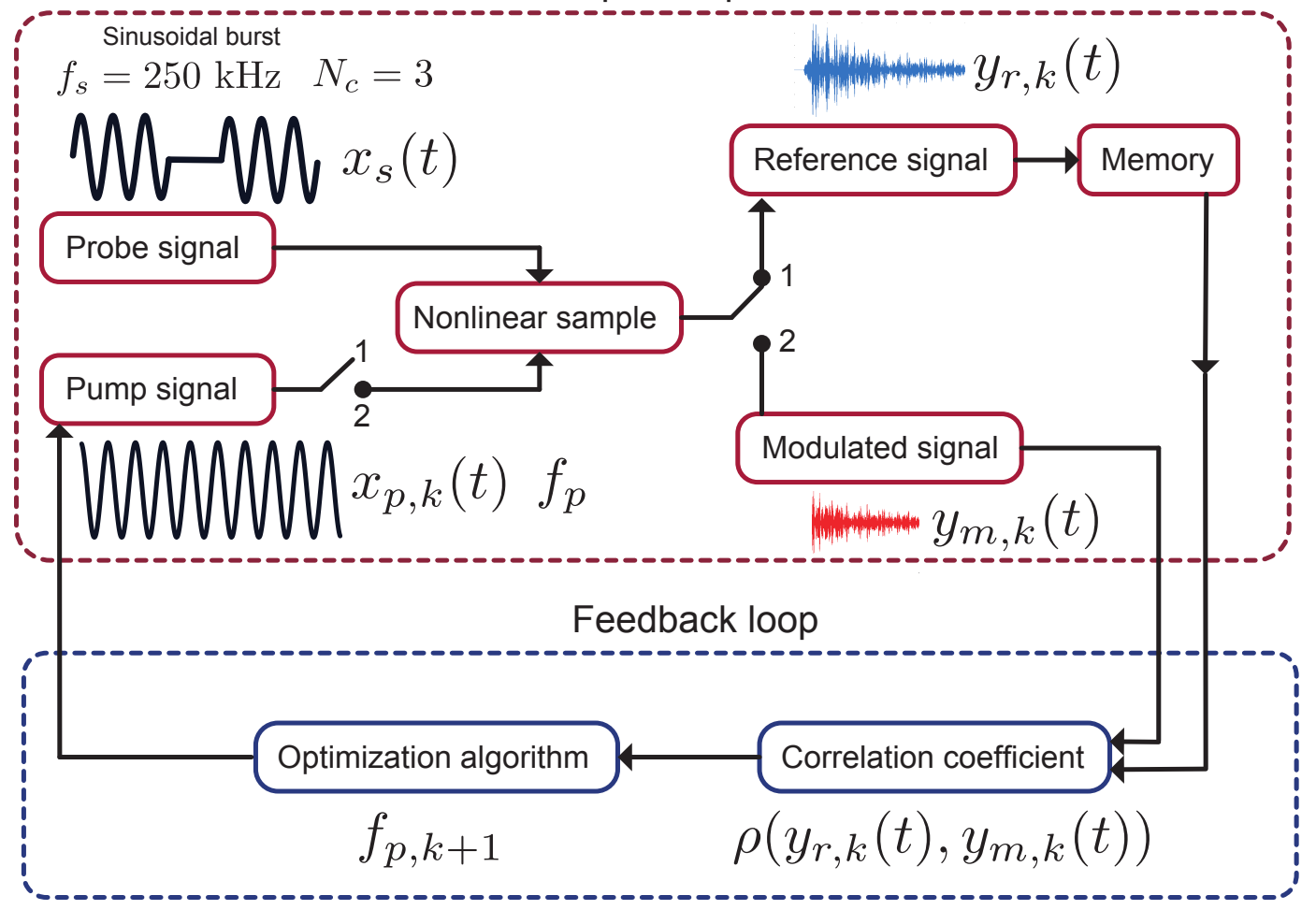

Figure 1: Bloc diagram of closed loop optimization of pump frequency for the VAM technique. The same probe excitation signal $x_{s}(t)$ is transmitted to the sample without the pumping excitation. The received reference probe coda signal $y_{r, k}(t)$ is recorded for a next use( switch position 1). In a second time (switch position 2), a pumping excitation $x_{p, k}(t)$ at a frequency $f_{p, k}$ is transmitted simultaneously with the probe excitation to the medium. The modulated coda probe signal $y_{m, k}(t)$ is also recorded. The added feedback consists of evaluating and minimizing the correlation coefficient $\rho$ between $y_{r, k}(t)$ and $y_{m, k}(t)$. The optimization algorithm allowed to find a new pumping frequency $f_{p, k+1}$.

effects. Usually, to quantify the resulting NM effects in the context of multiple scattering media, the Coda Wave Interferometry (CWI) in the time domain is used $[13,14,39,40]$. We have chosen to proceed similarly, and extract the nonlinear modulation information in the time domain as it seems 
to be more accessible. Indeed, the frequency response is very complex in multiple scattering media, and the modulation sidebands are difficult to see (see section 3.3).

The correlation coefficient $\rho$ between the received probe coda signal without pumping and with the pump excitation constitutes our cost function. With the presence of a nonlinear scatterer in the sample, the probe signal is expected to be modulated by the pump excitation which induces to a decorrelation between the two signals. A correlation coefficient equal to unity means that there is no influence of the pump on the probe wave, i.e., no nonlinear modulation effect in the sample. On the contrary, for the same pump amplitude value, a deviation of $\rho$ from 1 indicates the presence of nonlinear damage leading to a nonlinear modulation effect. In a theoretical point of view, the problem consists in calculating:

$$
f_{p}^{*}=\arg \min _{f_{p}}\left(\rho\left(f_{p}\right)\right) .
$$

During the optimization process, for each iteration $k$, the same probe excitation signal $x_{s}(t)$ is transmitted to the sample without the pumping signal (see Fig.1 switch position 1), and the received reference probe coda signal $y_{r, k}(t)$ is recorded for a next use. In a second time (switch position 2), a pump excitation $x_{p, k}(t)$ at a frequency $f_{p, k}$ is transmitted simultaneously with the probe excitation to the medium. The modulated coda probe signal $y_{m, k}(t)$ is also recorded. The added feedback consists in evaluating and optimizing a cost function, which is, in our case, the correlation coefficient between the two recorded received signals $\rho\left(y_{r, k},(t), y_{m, k}(t)\right)$ within the time interval 
122

$\left[t_{1}, t_{2}\right]$ such as:

$$
\rho\left(y_{r, k}(t), y_{m, k}(t)\right)=\frac{\int_{t_{1}}^{t_{2}}\left(y_{r, k}(t)-\bar{y}_{r, k}(t)\right)\left(y_{m, k}(t)-\bar{y}_{m, k}(t)\right) \mathrm{d} t}{\sqrt{\int_{t_{1}}^{t_{2}}\left(y_{r, k}(t)-\bar{y}_{r, k}(t)\right)^{2} \mathrm{~d} t \int_{t_{1}}^{t_{2}}\left(y_{m, k}(t)-\bar{y}_{m, k}(t)\right)^{2} \mathrm{~d} t}}
$$

where $\bar{y}_{r, k}(t)$ is the average of the reference signal within the interval $\left[t_{1}, t_{2}\right]$, and $\bar{y}_{m, k}(t)$ that of the modulated signal. This coefficient quantifies the resemblance between the reference probe signal and the modulated probe signal by the pump signal. The higher the nonlinear modulation between the probe and the pump signals is, the lower the value of the correlation coefficient is. An optimization algorithm is required to find a new pumping frequency $f_{p, k+1}$, at the iteration $k+1$, which maximizes the NM effect and therefore minimizes the correlation coefficient. The frequency is then modified and all the process described above is reiterated until the algorithm converges toward the best solution. In our study, the genetic algorithm was applied.

\subsection{Genetic Algorithm}

To find iteratively the pumping frequency giving the global minimum of the correlation coefficient, the genetic algorithm is used [41]. It is a search optimization technique based on the principles of genetics and natural selection. The genetic algorithm allows a population composed of a set of pumping frequencies to evolve under specified selection rules to a state that maximizes the "fitness" (i.e., minimizes the correlation coefficient)[34, 42]. The first step (called generation 1) consists of choosing randomly $\mathrm{N}$ pumping frequencies from a uniform distribution on a given frequencies interval. In our case, we have chosen $10 \mathrm{~Hz} \leq f_{p} \leq 900 \mathrm{~Hz}$. This choice is directly related 
to the empirical optimization for a comparison purpose. Indeed, a largest initial population could been chosen. The correlation coefficient is evaluated for each pumping frequency value and sorted in descending order. To prepare the next step, the $\mathrm{N} / 2$ best pumping frequencies that minimize the correlation coefficient are kept for the next generation $k+1$ and become parents. $\mathrm{N} / 2$ new pumping frequencies named offspring are generated following the expressions $[41,42]$ :

$$
\begin{aligned}
& \text { offspring } 1=f_{p m}-\beta\left[f_{p m}-f_{p d}\right] \\
& \text { offspring } 2=f_{p m}+\beta\left[f_{p m}-f_{p d}\right]
\end{aligned}
$$

where $\beta$ is a random value between 0 and 1 . the subscripts $m$ and $d$ discriminates between the mum and the dad pumping frequencies. A percentage of the samples is mutated to obtain a robust optimization. There are some algorithm parameters that must be chosen such the population size $\mathrm{N}$ and the mutation rate $\mathrm{R}$. In our case, $\mathrm{N}=12$ and the mutation rate $\mathrm{R}=40 \%$ [34]. Finally, after cost function evaluation, the pumping frequency with the lower correlation coefficient is the best solution of generation $k+1$. The genetic algorithm is adapted to global optimization problem; it means that it will be able to find the global minimum even if the function presents local minima. The GA is good for at identifying promising area of the search space but less efficient at fine-tuning the approximation to the minimum [43].

\section{Material}

\subsection{Sample description}

An aluminium bar $(600 \mathrm{~mm} \times 15 \mathrm{~mm} \times 3 \mathrm{~mm})$, with density $\rho=2700$ $\mathrm{kg} / \mathrm{m}^{3}$, Poisson ratio $\sigma=0.33$, and Young modulus E=69 GPa [44], is used as 
the specimen for our experiments. The probe wave propagation velocity in the bulk of the bar is estimated to $4838 \mathrm{~m} / \mathrm{s}$. 10 tapped holes with a $4 \mathrm{~mm}$ diameter and localized at distances of $10 \mathrm{~mm}, 30 \mathrm{~mm}, 50 \mathrm{~mm}, 100 \mathrm{~mm}$ and $200 \mathrm{~mm}$ from each sides of the bar center are drilled (see Fig. 2 from S1 to S10 from left to right). Identical screws $(\mathrm{m}=4.42 \mathrm{~g})$ can be placed in the tapped holes to mimic nonlinear solid contacts (cracks) such as in [45, 39, 40, 46]. These holes constitute linear scatterers when no screw is present. According to the number of nonlinear scatterers on the bar, different levels of "effective" damage can be obtained.

In the present study, three configurations have been more particularly studied: the first one (Config 1) corresponds to the case where no screw is placed in the sample bar; the reference. Nevertheless, one fixation screw is placed in the middle to insure the link between the shaker and the bar (S0 in Fig. 2). Configuration 2 (Config 2) corresponds to the case where only 2 screws are positioned at S2 and S9, and a third configuration (Config 3) is the case where all screws are placed (at S1, S2, S3, S4, S5, S6, S7, S8, S9, and S10). Note that the nonlinearity level in the sample depends on the screw number and the nuts tightening.

\subsection{Experimental Setup}

The closed loop pump frequency optimization process requires the experimental setup depicted in Fig. 3. Two broadband piezoelectric transducers with $250 \mathrm{kHz}$ central frequencies for transmitting and receiving the probe signal are glued to the ends of the sample. For the transmission of the probe signal, a $100 \mathrm{mVpp}$ sinusoidal burst of 3 periods of $250 \mathrm{kHz}$ frequency, repeated every 20 ms, was emitted by a function generator (AFG3022, Tektronix, 

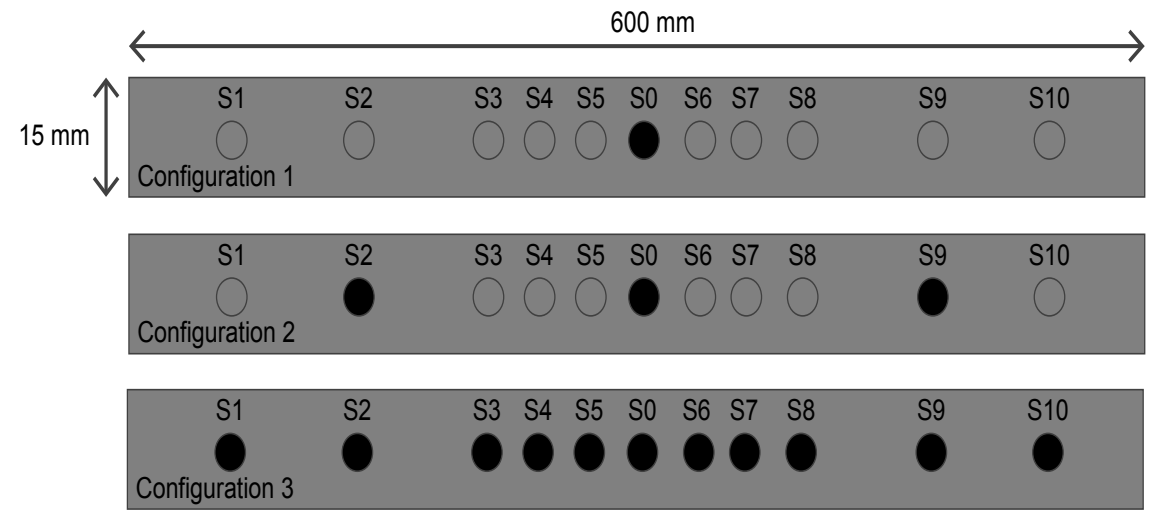

Figure 2: Specimen schemes of the three configurations. One Aluminium bar $(600 \mathrm{~mm}$ $\times 15 \mathrm{~mm} \times 3 \mathrm{~mm}$ ) containing 10 tapped holes. Config 1: no screws are placed. Config 2: 2 screws are placed at S2 and S9. Config 3: all the screws are placed on the bar.

Beaverton, Oregon, USA) and amplified to $60 \mathrm{~dB}(100 \mathrm{Vpp})$ by a power amplifier (Type 2713, Bruël \& Kjær, Nærum, Danemark). Simultaneously, a lower frequency continuous sine pump signal is generated by the computercontrolled function generator (Tektronix, AFG3021C, Beaverton, Oregon, USA) to change the excitation frequency during the closed-loop optimization process. The pump signal is amplified by a power amplifier (PA100E, Bruël \& Kjær, Nærum, Danemark), and transmitted to the shaker (LDS V406, Bruël \& Kjær, Nærum, Danemark) which is connected to the center of the sample by a screw. The coda probe signal is detected by the receiving transducer and amplified by a preamplifier (Ciprian, Saint ISMIER, France), then, transmitted to an oscilloscope (LT 264ML, Lecroy, Chestnut Ridge, NY, USA). In order to improve the signal to noise ratio, an average of 300 successive acquisitions is carried out, and a coda averaged signal is recorded. Each measurement lasts about 10s. Both the function generator and the oscilloscope are controlled by MATLAB (Mathworks, Natick, MA, USA). 

over the acquired signal. By comparison, An experimental modal analysis fects are correctly distributed over the successive acquisitions and averaged

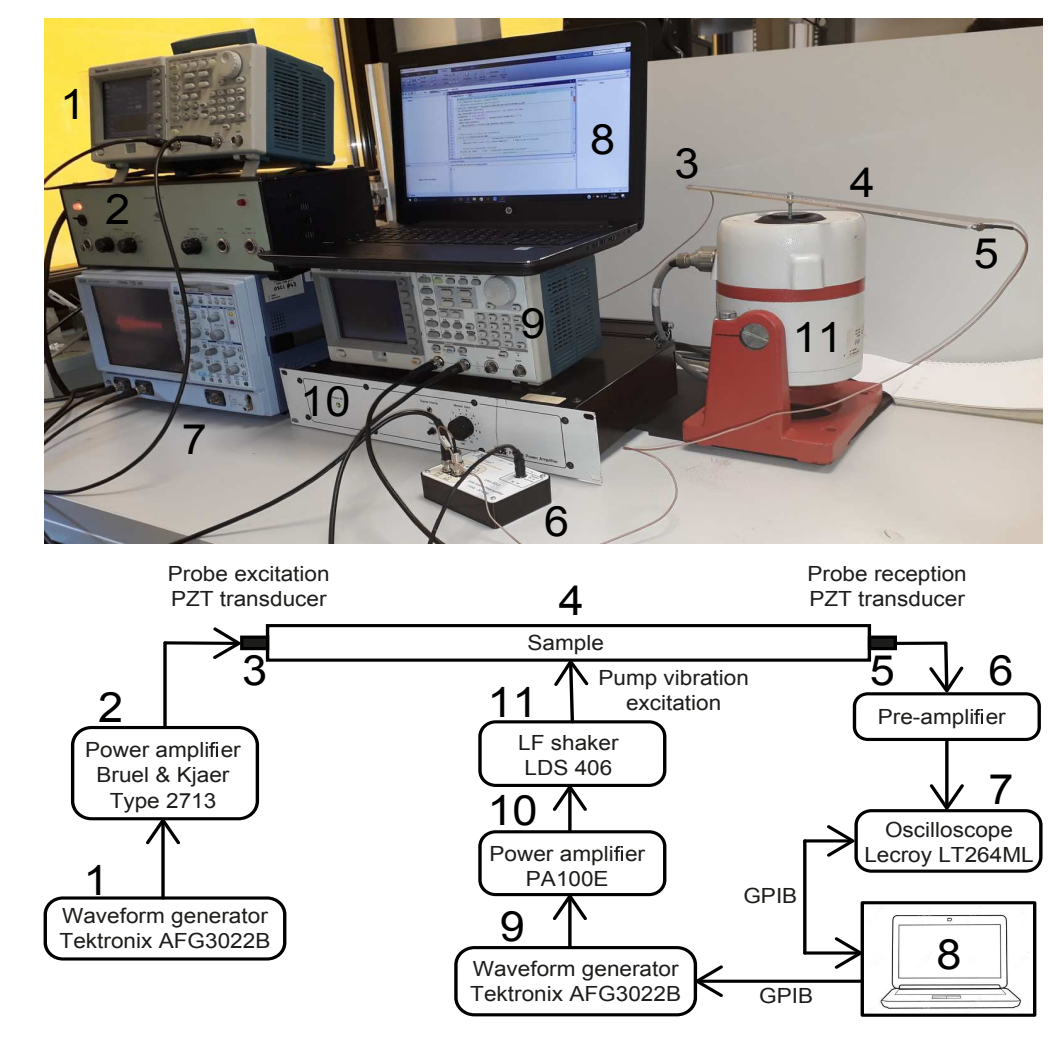

Figure 3: Experimental setup for the closed loop Vibro-Acoustic Modulation (VAM) technique.

The pump signal is desynchronized from the probe so that the nonlinear ef(2)

is achieved to identify the frequencies of the first structural resonances of the sample. For this purpose, a frequency swept sine signal from $5 \mathrm{~Hz}$ to $1000 \mathrm{~Hz}$ is generated using a signal analyzer (SR785, Stanford Research Systems Inc., Sunnyvale, CA, USA). The signal is amplified by a PA100E power amplifier ,then, transmitted to a LDS406 shaker. The data are acquired by an accelerometer (352C23, PCB Piezotronics Inc, Depew, NY, USA) then 
transmitted to the signal analyzer. Both the input excitation and the output response are used to obtain the averaged Frequency Response Function (FRF) of the sample. The excitation and the acquisition are controlled by a GPIB connection via MATLAB.

\subsection{Nonlinear modulation effects on the probe coda signal}

In order to show the NM effects on the temporal coda signal, two configurations are considered. The first one corresponds to the case without screws (Config 1), and the second one corresponds to the Config 2. The pump frequency is $150 \mathrm{~Hz}$. Fig. 4 shows the received probe coda signal from the Config 1 (Fig. 4a) and Config 2 (Fig. 4d). For each case, early and late temporal windows are shown with and without the presence of the pump excitation (Figs. 4b, c and e, f). The pump presence causes no detectable change on the probe coda signal obtained from the intact sample, for early and late windows: there is no nonlinear modulation of the probe by the pump with a correlation coefficient of 1 . On the contrary, for the damaged sample, it can easily be noted that the two coda signals recorded with and without the pumping wave are different for the late window $\mathrm{t}=[1,1.05] \mathrm{ms}$ (Fig. 4f), with a correlation coefficient $\rho=0.976$. It indicates that a NM has occurred between the probe and the pump waves. Moreover, the nonlinear effects are more visible in the late window because, at this time, the wave has crossed the medium and interacted with the nonlinear scatterers several times. From previous implementations of the nonlinear modulation of a coda wave by a pump wave in a multiple scattering medium $[13,14,46]$, the decorrelation is either due to a localized velocity variation, to an amplitude- dependent dissipation effect or to an amplitude-dependent scattering effect. By introducing 
internal solid contacts in the medium, between the screws and the holes, amplitude- dependent clapping, tapping and frictional effects are expected. These results consolidate our choice of quantifying the nonlinear modulation effects by calculating the correlation coefficient between the probe coda signals obtained with and without the presence of the pump excitation, in a time interval between $t_{1}=1 \mathrm{~ms}$ and $t_{2}=1.2 \mathrm{~ms}$. It is important to notice that the choice of the central time and the width of the temporal window in which the correlation coefficient is estimated, obeys to some constraints [47]. The characteristic scattering time $\mathrm{t}^{*}$ is estimated according to the method proposed in [48], and is find to be around $3 \mu$ s for our specimen. The starting time of our window $t_{1}=1 \mathrm{~ms}$ satisfies the condition $t_{1} \gg \mathrm{t}^{*}$. This ensures a global inspection of the entire studied sample. Moreover the time interval width is set to $200 \mu \mathrm{s}$, such as it includes enough signal periods (50 periods of the probe signal at $250 \mathrm{kHz}$ ). The choice of the window position is also limited by the signal quality. Indeed, the more $t$ is increased, the more the signal to noise ratio is degraded and the higher the uncertainty on the result is.

In parallel, we illustrate in Fig. 5, the frequency spectra (right) of the coda probe signals (left), obtained for Config 2, with and without the presence of the pump wave excitation at $f_{p}=150 \mathrm{~Hz}$. The selected time window is $\Delta \mathrm{t}=$ $[1,1.2] \mathrm{ms}$. We can notice that both frequency spectra are centered on 250 $\mathrm{kHz}$, and include peaks due to the multiple scattered probe signal. The modulation sidebands are difficult to see in this case, and the only difference between the two spectra is an amplitude variation and a slight frequency shift. Moreover, the frequency resolution is not sufficient to observe the 

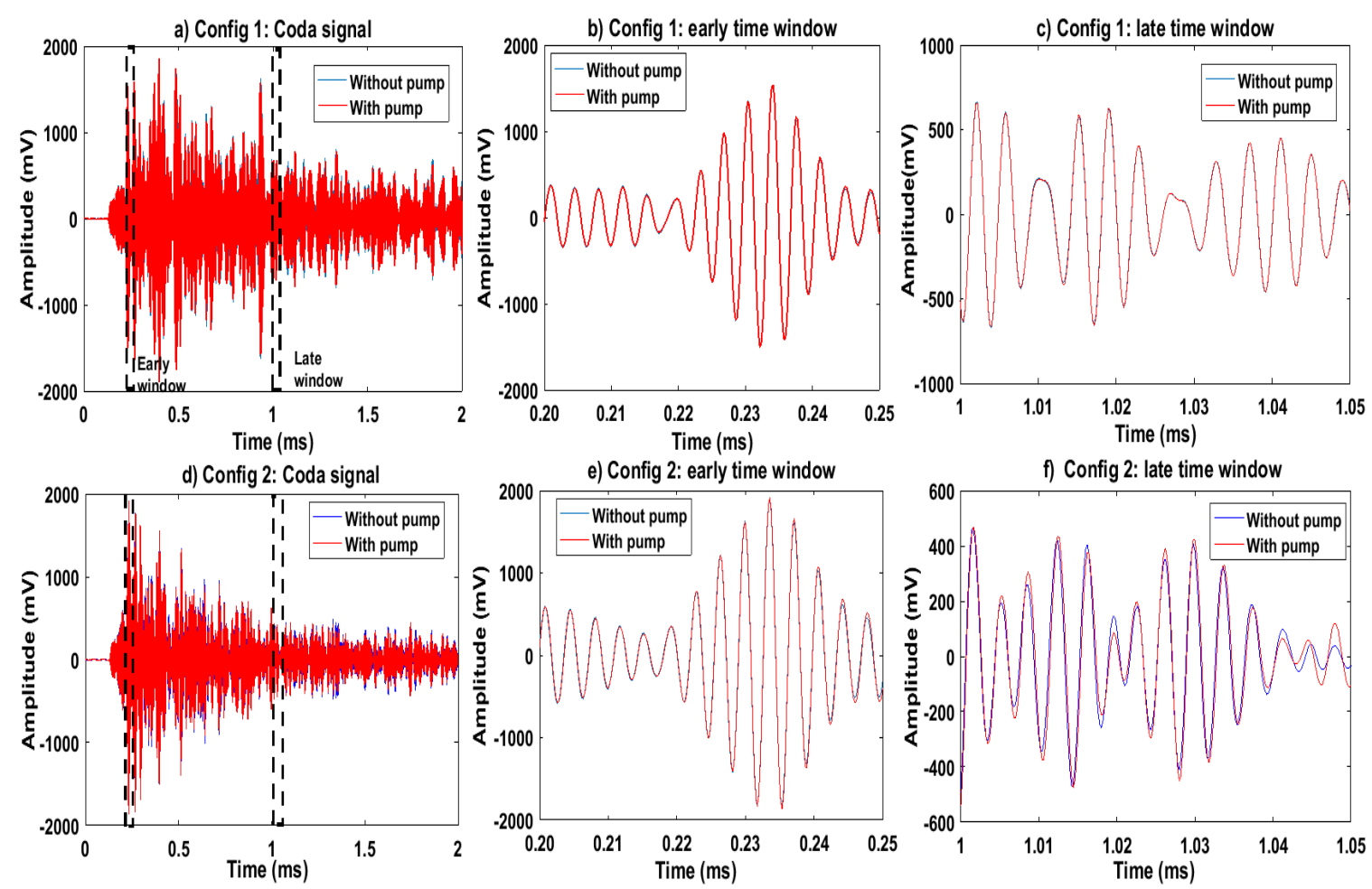

Figure 4: Experimental temporal coda probe signals obtained from the Config 1 (without screws): (a), (b) and (c), and for the Config 2 (with screws): (d), (e) and (f), with and without the pump excitation. The pump excitation is a continuous sine signal at a resonance frequency $f_{p}=150 \mathrm{~Hz}$. (b) and (e) are captures of the coda signals (a) and (d), respectively, during an early time interval between $[0.2,0.25] \mathrm{ms}$. (c) and (f) are captures of (a) and (d), respectively, during a late interval between [1, 1.05] ms.

modulation sidebands when $f_{\text {pump }} \ll f_{\text {probe }}$. Therefore, we choose to extract to modulation effects information in the time domain.

During all this study, it should be noticed that the decorrelation values are not observed higher than $2.5 \%$. We recall, here, that we want to test our approach in a rather unfavorable configuration, i.e., when the variation of 

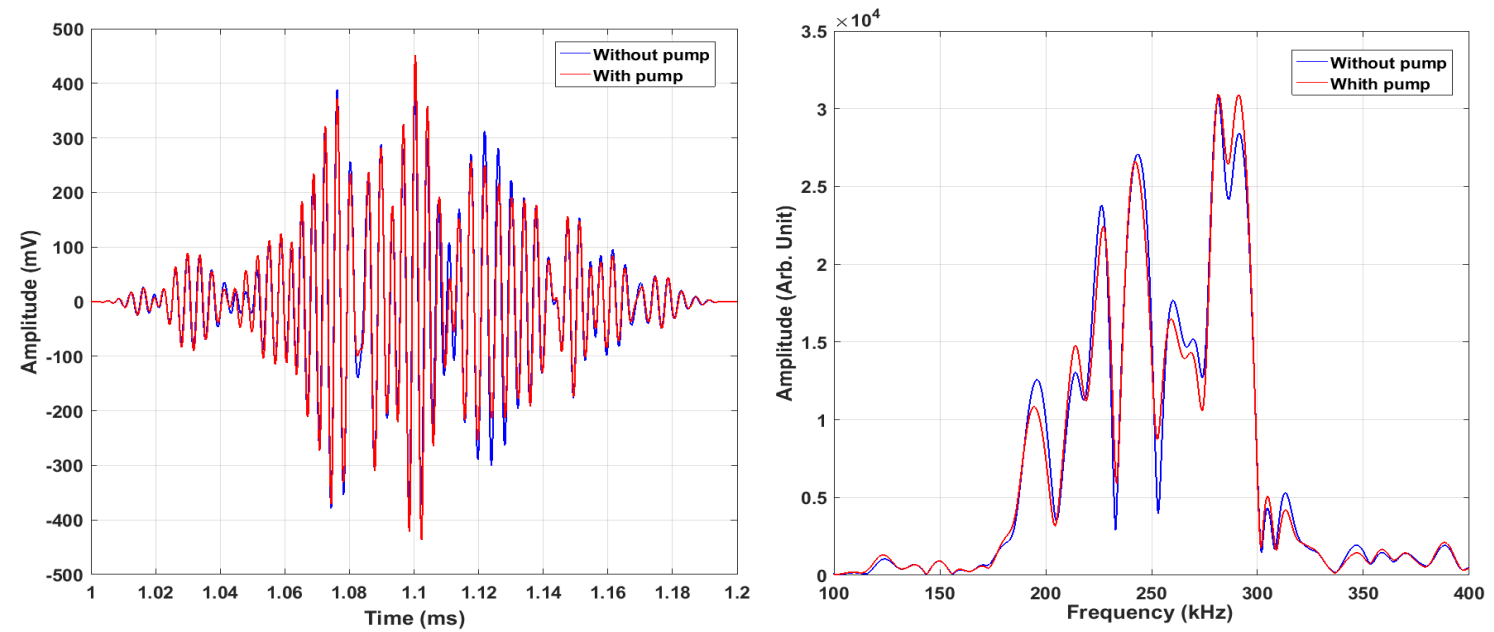

Figure 5: (left) Experimental probe coda signals obtained from the Config 2, with and without the presence of the pump. The pump excitation is a continuous sine wave at $150 \mathrm{~Hz}$. The coda signal is filtered with a hanning window for a duration $\Delta \mathrm{t}=[1,1.2] \mathrm{ms}$. (right) The frequency spectra of the coda signals.

the correlation coefficient is small. Indeed, a stronger nut tightening and a higher pump amplitude would have given higher levels of decorrelation.

\section{Experimental Results}

In this section, the main experimental results are presented. First an experimental modal analysis is performed in order to obtain the resonance modes of the sample for a comparison purpose. Then, an empirical research of the correlation coefficient $\rho$ behavior versus the pump frequency is presented and compared to the modal analysis results. Finally, a closed loop optimization of the correlation coefficient by the genetic algorithm is performed and presented. 


\subsection{Empirical optimization: Correlation coefficient dependency on the pump} frequency

The frequency response function (FRF) is obtained using the experimental input and output data obtained from the experiment described in the section 3.2. This preliminary experiment allows identifying the frequency resonance modes of the sample in the Configs 2 and 3. Figs. $6 a$ and $6 \mathrm{c}$ show the FRFs obtained for Configs 2 and 3, respectively, in a frequency range from $5 \mathrm{~Hz}$ to $900 \mathrm{~Hz}$. We can see that the magnitude of the resonance peaks and the corresponding frequencies are different for the two configurations. The results show that there is a shift in the natural frequencies and an overall change in the frequency response due to the effect of the added screws.

The first performed experiment is to check the existence of global or local minima for the correlation coefficient versus the pump frequency. This step is called "empirical optimization". For time saving, a pump frequency sine sweeping from $10 \mathrm{~Hz}$ to $900 \mathrm{~Hz}$ with a step of $10 \mathrm{~Hz}$ is achieved. This allows a global view of the correlation coefficient dependency on the pump frequency. For this first pass, the prominent observation is the presence of local minima peaks localized at specific vibration frequencies corresponding well to the resonances of the bar, and $\rho \simeq 1$ elsewhere. To refine the results and to get a better accuracy, a second sweeping was achieved with a finer frequency step of $1 \mathrm{~Hz}$, over the frequency regions where $\rho$ deviates from 1 (Figs. 6b and 6d). The procedure cited in the section 2.1 is adopted to calculate the correlation coefficient for each pump frequency. As illustrated in Fig. 6, the pump frequencies that give minima peaks of $\rho$ coincide well 
with the resonance frequencies of the sample for the Configs 2 and 3. This confirms the expected effects that at a resonance frequency of the sample, the vibration amplitude is naturally amplified by constructive interferences, leading to a more efficient nonlinear modulation effect. Also, we can note a frequency shift to the lower frequencies with the increase of the number of screws in the plate, as it can be seen in Figs. 6b and 6d. Additionally, we can see clearly that for the same resonance mode, the correlation coefficient values vary from one configuration to another. In fact, the second minimum peak corresponding to $f_{p}=150 \mathrm{~Hz}$ is the most important for the Config 2; $\rho=0.975$ (Fig. 6b) while $\rho=0.981$ at $f_{p}=144 \mathrm{~Hz}$ for the Config 3 (Fig. 6d). We can also notice the third $\rho$ peak is very close to $1(\rho=0.998)$ for the Config 2 (Fig. 6b) and equal to 0.986 for the Config 3. These observations confirm the well-known fact that in the pump resonance configuration, the NM efficiency on the probe wave by the pump wave depends on the pumping frequency/mode and on the exact position of the nonlinear scatterers. Based on the obtained curves, it can be stated that for each configuration, it exists an optimal pump frequency that maximizes the nonlinear modulation effects and, thus, the damage detection sensitivity. This optimal frequency corresponds to the frequency giving the lowest minimum of $\rho$. The empirical experiment also shows that the correlation coefficient is a good indicator to evaluate the nonlinear wave mixing in the sample. For an easier comparison of results obtained from Fig. 6, table 1 summarizes the correlation coefficient minima peaks values and the corresponding pumping frequencies obtained from the empirical optimization (EO) and those obtained from the FRF for both configurations. 

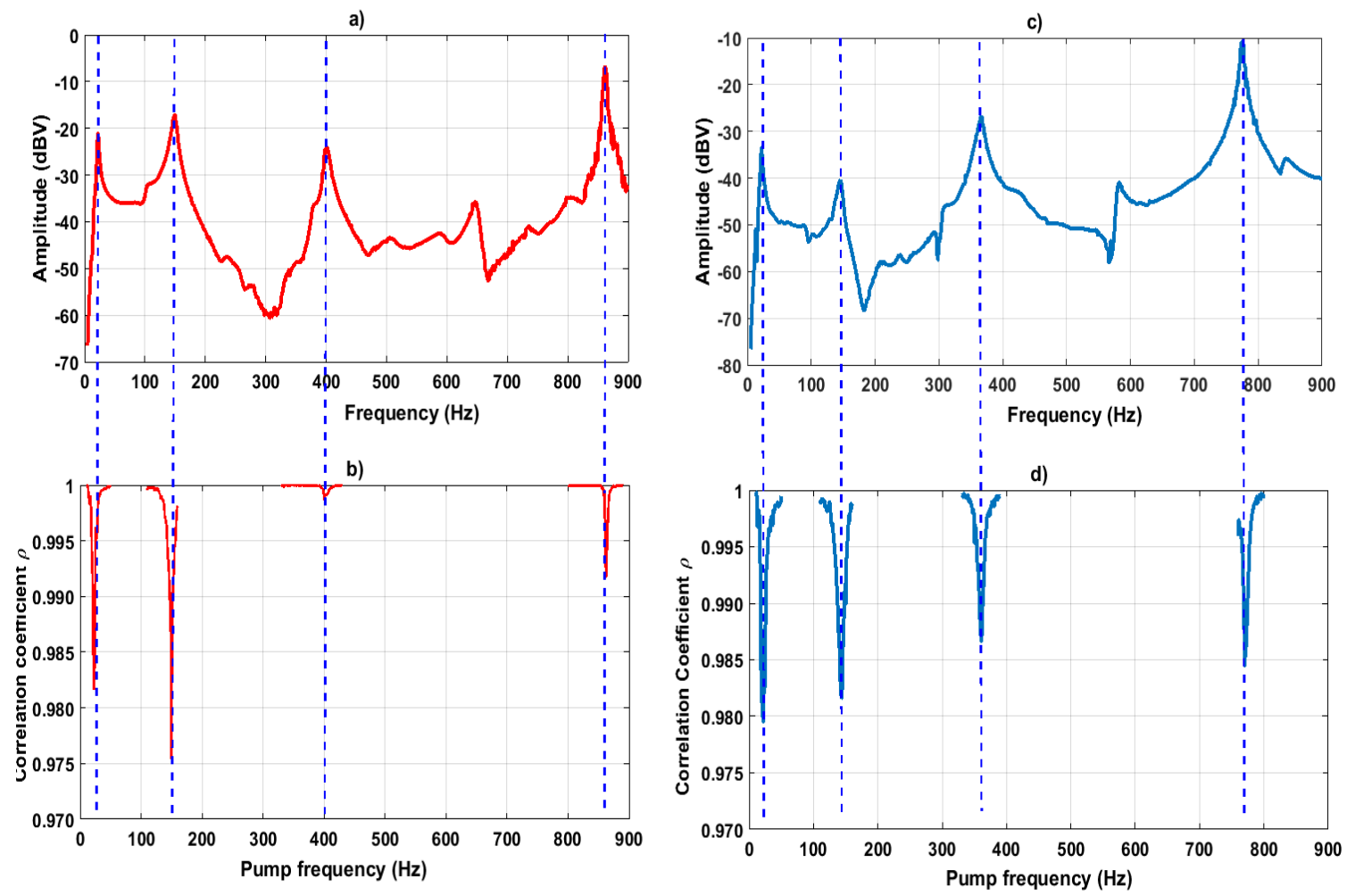

Figure 6: Comparison between the resonance frequencies obtained from the experimental FRF of the Config 2 (a) and the Config 3 (c). Experimental results of correlation coefficient versus pump frequency at the sample resonance frequencies areas for the two sample configurations: b) Config 2 and d) Config 3. The FRF peaks coincide with the minimum peaks of the correlation coefficient versus pumping frequency.

\subsection{Optimal pumping frequency excitation}

Fig. 7 shows the results of the pump frequency optimization for the Config 2 and Fig. 8 shows those obtained for the Config 3 when using the genetic algorithm optimization. For more clarity, the result of the empirical optimization is represented in Figs. 7a and 8a with a zoom on the GA covergence area. The initial population was included in a frequency range between $10 \mathrm{~Hz}$ 


\begin{tabular}{l|ccc|ccc}
\hline & \multicolumn{3}{|c|}{ Config 2 } & \multicolumn{3}{c}{ Config 3 } \\
Modes & $\rho_{E O}$ & $f_{p, E O}(\mathbf{H z})$ & $f_{p, F R F}(\mathbf{H z})$ & $\rho_{E O}$ & $f_{p, E O}(\mathbf{H z})$ & $f_{p, F R F}(\mathbf{H z})$ \\
\hline $1^{\text {st }}$ Mode & 0.981 & 23 & 22.1 & 0.979 & 22 & 22.1 \\
$2^{\text {nd }}$ Mode & 0.975 & 150 & 148.7 & 0.981 & 144 & 145.7 \\
$3^{\text {rd }}$ Mode & 0.998 & 400 & 401.0 & 0.986 & 361 & 365.8 \\
$4^{\text {th }}$ Mode & 0.991 & 862 & 861.3 & 0.984 & 770 & 773.9 \\
\hline
\end{tabular}

Table 1: The correlation coefficient $\rho$ and the $f_{p}$ values corresponding to the minimum peaks obtained from the empirical optimization (EO) and the resonance frequencies obtained from the Frequency Response Function (FRF) for Config 2 and 3.

and $900 \mathrm{~Hz}$, with a population size of 6 for the Config 2 and 12 for the Config 3. We can see that in both cases, the optimal pump frequency is reached after 4 generations for the Config 2 with an optimal pump frequency of 153.2 $\mathrm{Hz}$ and $\rho=0.991$ (Figs. 7b and 7c), and after 7 generations for the Config 3 with $f_{p}=22.17 \mathrm{~Hz}$ and $\rho=0.971$ (Figs. 8b and 8c). As it is illustrated in Figs. 7a and 8a the obtained results are quite similar with those obtained by the empirical optimization. For Config 2, the optimal pump frequency is reached by the genetic algorithm with a slight discrepancy in the pump frequency and the corresponding correlation coefficient. Indeed, we can see in Fig. 7a, that the GA converges nearly to the global minimum at $f_{p}=153.2 \mathrm{~Hz}$, in a pump frequency range between 10 and $900 \mathrm{~Hz}$, including four (4) local minima of the cost function, but the accurate value of this minimum, at $f_{p}=150 \mathrm{~Hz}$, is not reached. We suppose that a greater number of iterations should improve this result. Moreover, it is important to notice that for the empirical search, the step between two pump frequencies is chosen empirically, this may be 
(a)

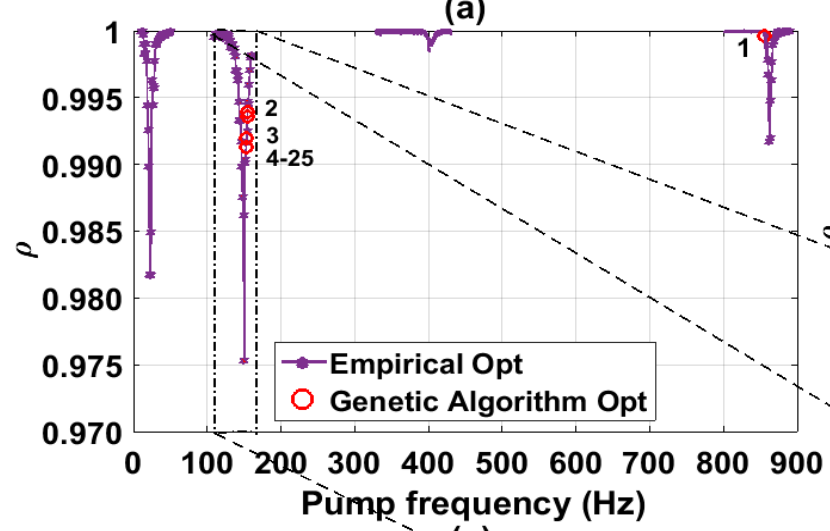

(c)

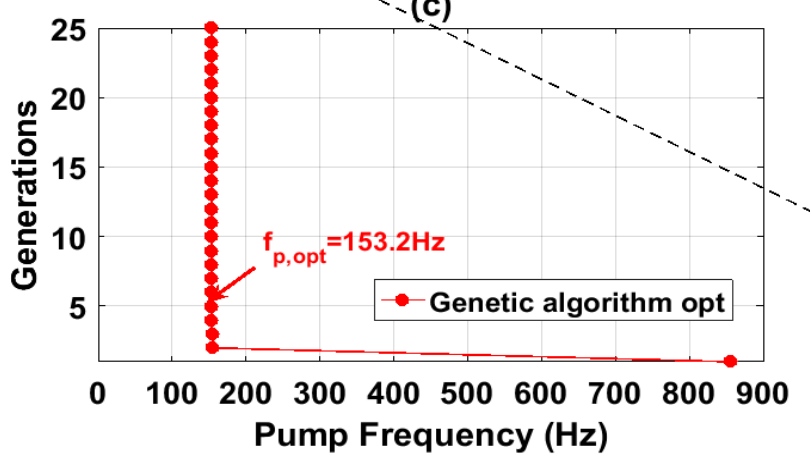

(b)

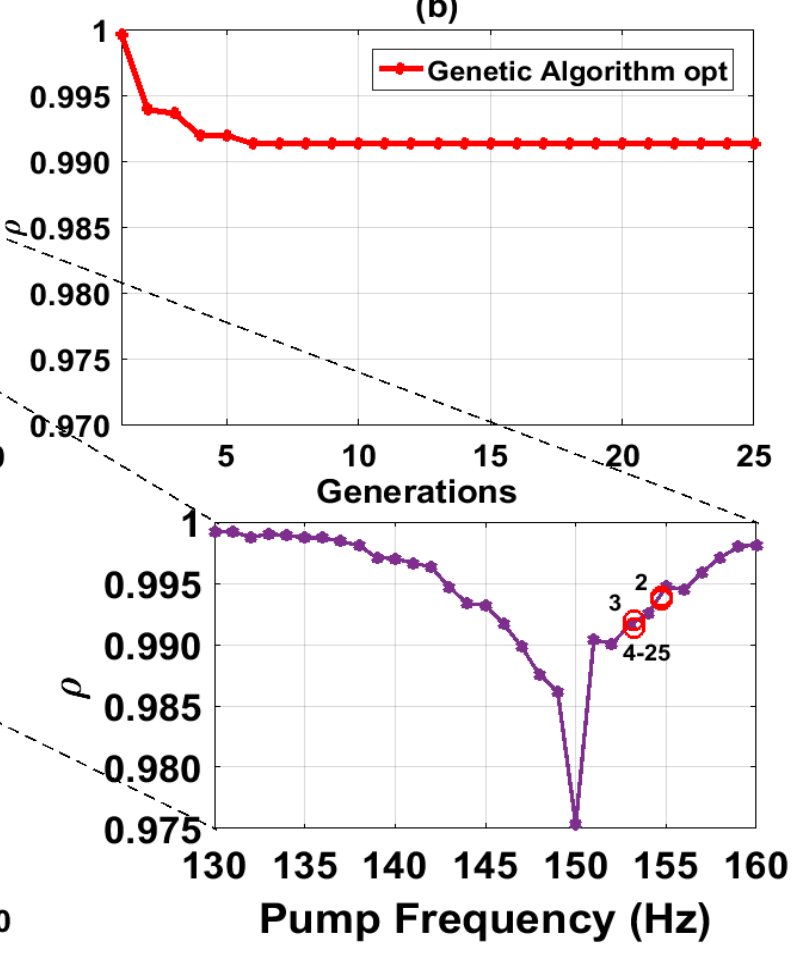

Figure 7: Genetic algorithm (GA) optimization results for the Config 2: (a) Empirical optimization and GA iteration numbers (red circles), a zoom on the GA convergence area is illustrated. (b) The correlation coefficient $\rho$ versus generations. (c) The corresponding pump frequency versus generations. The GA converges to $f_{p, o p t}=153.2 \mathrm{~Hz}$ after the $4^{\text {th }}$ generation.

the origin of the discrepancy observed between the empirical and the automatic optimization. For the Config 3, the optimal pump frequency is nearly reached. For both cases, the pump frequency converges while $\rho$ remains variable for the same value of the pump frequency. This can be clearly seen in Figs. $8 \mathrm{~b}$ and $8 \mathrm{c}$ where the pump frequency converges to $f_{p}=22.17 \mathrm{~Hz}$, and the corresponding $\rho$ still varies slightly. This observation is directly due to 
(a)

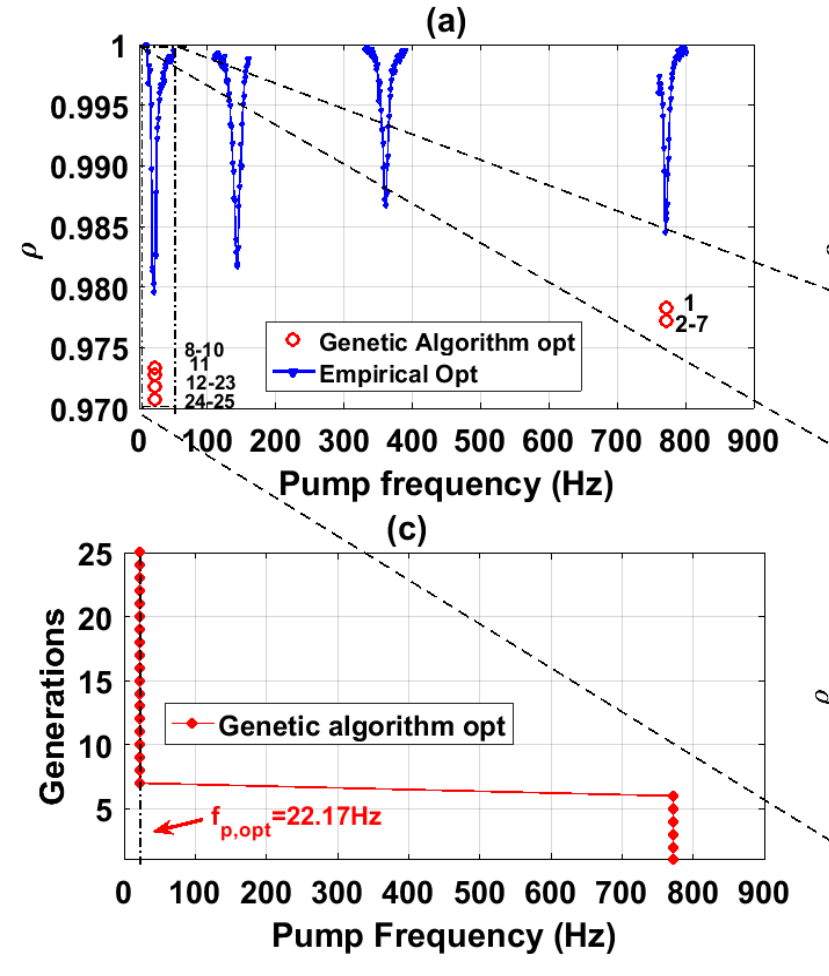

(b)
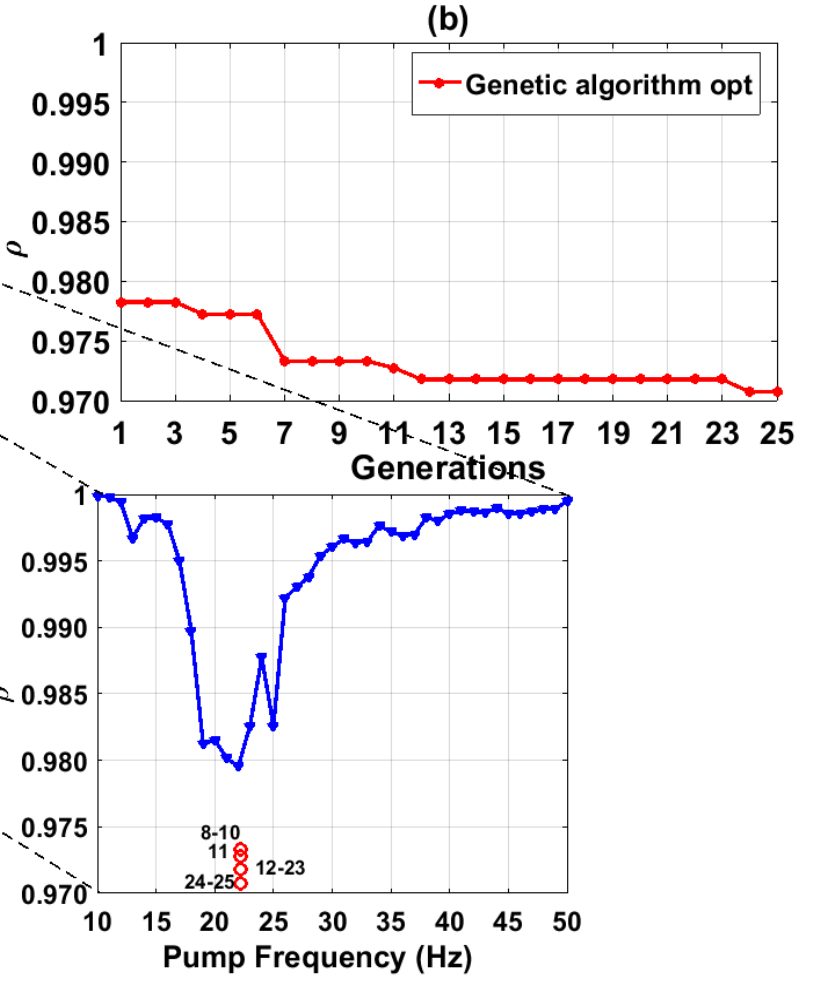

Figure 8: Genetic algorithm (GA) optimization results for the Config 3: (a) Empirical optimization and GA iteration numbers (red circles), a zoom on the GA convergence area is illustrated. (b) The correlation coefficient $\rho$ versus generations. (c) The corresponding pump frequency versus generations. The GA converges to $f_{p, o p t}=22.17 \mathrm{~Hz}$ after the $7^{\text {th }}$ generation.

the experimental conditions since the coda signal is very sensible to small temperature changes. Moreover, the correlation coefficient value reaches a lower value than that obtained by the empirical optimization (Fig. 8a). It is important to mention that the empirical cost function is not a continuous function, and a measurement is made with a pump frequency step of $1 \mathrm{~Hz}$. We think, that a finer pump frequency step between two successive cost function 


\begin{tabular}{l|cc|cc}
\hline & \multicolumn{2}{|c|}{ Config 3 } & \multicolumn{2}{c}{ Config 2 } \\
& $\rho$ & $f_{p, \text { opt }}(\mathbf{H z})$ & $\rho$ & $f_{p, o p t}(\mathbf{H z})$ \\
\hline Genetic Algorithm & 0.971 & 22.17 & 0.991 & 153.2 \\
Empirical optimization & 0.979 & 22.00 & 0.975 & 150.0 \\
\hline
\end{tabular}

Table 2: Experimental optimization results of the genetic algorithm covergence and the empirical values of the global minimum obtained by the empirical search, for the Config 2 and 3.

measurements should give a better concordance between the empirical and the automatic search by the GA. Another possible explanation is that for the empirical optimization, one measurement of the cost function has been made for each pump frequency value between $10-900 \mathrm{~Hz}$, while for the GA optimization, and after convergence, several measurements have been made at the same optimal pump frequency (resonance frequency). In fact, exciting a sample at its resonance frequency during a long time (conditioning), could gives rise to a greater decorrelation between the reference coda signal and the modulated coda signal. Indeed, for the modal excitation, the vibration response is amplified such as the medium does not reach the equilibrium and the reference signal is still affected by the previous iterations. This may be linked to "slow dynamics" effects [49].

We can also note that the algorithm convergence is faster when the population size is lower. The strong point of our approach is that the algorithm converged to the global minimum of the correlation coefficient without a priori information. For more clarity, table 2 summarizes the results obtained by both the empirical and the GA optimization. 


\section{Discussion and Conclusion}

In this work, a widely studied nonlinear ultrasonic nondestructive testing method has been combined with the optimal command method: an open loop Vibro Acoustic Modulation system has been enriched with a feedback system. The main idea was to achieve an optimal damage detection by finding automatically the best input pump frequency. This input frequency maximizes the nonlinear modulation effects induced by the presence of nonlinear scatterers and, therefore, should increase the sensitivity of the VAM method. The best pump frequency adjusted to each configuration has been successfully found by the feedback method. This method is based, principally, on the good choice of a so called "cost function" and the optimization parameter. Moreover, the experiment setting is easy and not user dependent. The genetic algorithm was tested on two sample configurations with different levels of nonlinearity to demonstrate the adaptivity of the proposed method. It is found able to determine automatically the optimal pump frequency over a wide frequency range including 4 resonance modes. Even if the genetic algorithm is not always efficient to find the accurate optimal value, it guarantees to find the global optimum region quite fast since four (4) generations were sufficient in our case. A preliminary empirical search was achieved to check for the behavior of the cost function, the correlation coefficient as a function of the optimization parameter (the pump frequency). The results of this empirical search, requiring a long blind experimental search, have been presented in the form of correlation coefficient versus pump frequency. The main outcome is the existence of frequencies, which correspond to some of the resonance frequencies of the sample, giving a maximum of decorrelation (or 
a minimum correlation). It constitutes a first suitable result which confirms our hypothesis of choosing the correlation coefficient of the probe coda signal, with and without pumping to quantify the NM effects, and the pumping frequency as an optimization parameter. The quantitative variation of $\rho$ with the pump resonance frequencies, for the different configurations is related to the modal vibration shapes and the associated strain amplitude changes from a resonance mode to another, at the location of the nonlinear scatterers. For different arrangements of nonlinear scatterers, we observe differences in the values of the correlation coefficient, because a given resonant mode does not necessarily excites differently located nonlinear scatterers with the same efficiency. Similarly, for a given medium configuration, but when comparing two different resonant modes, the nodes and anti-nodes positions are not located identically, and they do not excite with the same efficiency a given nonlinear scatterer. As such, using a single pump frequency leads to the apparition of blind zones for the VAM, e.g. when a single scatterer is at a strain node. For this reason, a preliminary modal analysis to determine a resonance modes of the studied medium seems to be not sufficient to ensure an increase of the VAM sensitivity detection.

The proposed method, in a resonance configuration, ensures to avoid the low sensitivity regions associated with the strain nodes, which could compromise the nonlinear scatterer detection. Moreover, for a fixed pump amplitude, the adjusted pump frequency excitation may bring maximum detection sensitivity for nonlinear damages, which can be very useful to detect early stage damage in materials. Furthermore, the advantage of the automatic optimization is a time gain compared to the empirical optimization which 
takes about 15 times more then our method.

The optimal command can be extended to other NDT methods by defining the right cost functions adapted to the optimization purpose. Also, as a perspective, the genetic algorithm outcomes could be used as the initialization for a gradient descent algorithm in order to refine the results. In addition, information on the local minima of the correlation coefficient may be relevant for locating the nonlinear scatterers: each resonant mode constitutes a spatial sensitivity kernel for the method and information stacking for several modes may offer imaging capabilities.

\section{References}

[1] O. Buck, W. Morris, J. M. Richardson, Acoustic harmonic generation at unbonded interfaces and fatigue cracks, Applied Physics Letters 33 (5) (1978) 371-373.

[2] I. Y. Solodov, Ultrasonics of non-linear contacts: propagation, reflection and nde-applications, Ultrasonics 36 (1-5) (1998) 383-390.

[3] P. B. Nagy, Fatigue damage assessment by nonlinear ultrasonic materials characterization, Ultrasonics 36 (1-5) (1998) 375-381.

[4] Y. Zheng, R. G. Maev, I. Y. Solodov, Review/sythèse nonlinear acoustic applications for material characterization: a review, Canadian Journal of Physics 77 (12) (2000) 927-967.

[5] K.-A. Van Den Abeele, J. Carmeliet, J. A. Ten Cate, P. A. Johnson, Nonlinear elastic wave spectroscopy $(N E W S)$ techniques to discern material 
damage, part II: Single-mode nonlinear resonance acoustic spectroscopy, Journal of Research in Nondestructive Evaluation 12 (1) (2000) 31-42.

[6] V. V. Kazakov, A. Sutin, P. A. Johnson, Sensitive imaging of an elastic nonlinear wave-scattering source in a solid, Applied Physics Letters 81 (4) (2002) 646-648.

[7] G. Renaud, S. Callé, M. Defontaine, Remote dynamic acoustoelastic testing: Elastic and dissipative acoustic nonlinearities measured under hydrostatic tension and compression, Applied Physics Letters 94 (1) (2009) 011905.

[8] D. M. Donskoy, A. M. Sutin, Vibro-acoustic modulation nondestructive evaluation technique, Journal of intelligent material systems and structures 9 (9) (1998) 765-771.

[9] L. Pieczonka, A. Klepka, A. Martowicz, W. J. Staszewski, Nonlinear vibroacoustic wave modulations for structural damage detection: an overview, Optical Engineering 55 (1) (2015) 011005.

[10] K.-A. Van Den Abeele, P. A. Johnson, A. Sutin, Nonlinear elastic wave spectroscopy $(N E W S)$ techniques to discern material damage, part $I$ : nonlinear wave modulation spectroscopy $(N W M S)$, Journal of Research in Nondestructive Evaluation 12 (1) (2000) 17-30.

[11] M. Meo, U. Polimeno, G. Zumpano, Detecting damage in composite material using nonlinear elastic wave spectroscopy methods, Applied composite materials 15 (3) (2008) 115-126. 
[12] G. Gao, D. Li, D. Shi, J. Dong, X. Shi, F. Teng, Nonlinear acoustic characteristics of fatigue microcracks in al alloy plate, JOM 63 (2) (2011) $77-80$.

[13] Y. Zhang, V. Tournat, O. Abraham, O. Durand, S. Letourneur, A. Le Duff, B. Lascoup, Nonlinear mixing of ultrasonic coda waves with lower frequency-swept pump waves for a global detection of defects in multiple scattering media, Journal of Applied Physics 113 (6) (2013) 064905.

[14] Y. Zhang, V. Tournat, O. Abraham, O. Durand, S. Letourneur, A. Le Duff, B. Lascoup, Nonlinear coda wave interferometry for the global evaluation of damage levels in complex solids, Ultrasonics 73 (2017) 245-252.

[15] H. Hu, W. Staszewski, N. Hu, R. Jenal, G. Qin, Crack detection using nonlinear acoustics and piezoceramic transducers instantaneous amplitude and frequency analysis, Smart materials and structures 19 (6) (2010) 065017.

[16] A. Klepka, W. Staszewski, R. Jenal, M. Szwedo, J. Iwaniec, T. Uhl, Nonlinear acoustics for fatigue crack detection-experimental investigations of vibro-acoustic wave modulations, Structural Health Monitoring 11 (2) (2012) 197-211.

[17] L. Pieczonka, A. Klepka, M. Adamczyk, W. Staszewski, F. Aymerich, T. Uhl, Optimal selection of parameters for impact damage detection in composites based on nonlinear vibro-acoustics modulations, in: 
ECCM16 16th European Conference on Composite Materials, Seville, Spain, 22-26 June 2014.

[18] B. Liu, Z. Luo, T. Gang, Influence of low-frequency parameter changes on nonlinear vibro-acoustic wave modulations used for crack detection, Structural Health Monitoring (2017) 1475921716689385.

[19] N. C. Yoder, D. E. Adams, Vibro-acoustic modulation utilizing a swept probing signal for robust crack detection, Structural Health Monitoring 9 (3) (2010) 257-267.

[20] H. Sohn, H. J. Lim, M. P. DeSimio, K. Brown, M. Derriso, Nonlinear ultrasonic wave modulation for online fatigue crack detection, Journal of Sound and Vibration 333 (5) (2014) 1473-1484.

[21] B. Liu, T. Gang, C. Wan, C. Wang, Z. Luo, Analysis of nonlinear modulation between sound and vibrations in metallic structure and its use for damage detection, Nondestructive Testing and Evaluation 30 (3) (2015) 277-290.

[22] K. Dziedziech, L. Pieczonka, M. Adamczyk, A. Klepka, W. J. Staszewski, Efficient swept sine chirp excitation in the non-linear vibroacoustic wave modulation technique used for damage detection, Structural Health Monitoring 17 (3) (2018) 565-576.

[23] M. Dunn, A. Carcione, P. Blanloeuil, M. Veidt, Critical aspects of experimental damage detection methodologies using nonlinear vibroultrasonics, Procedia Engineering 188 (2017) 133-140. 
[24] C. R. Courtney, B. W. Drinkwater, S. A. Neild, P. D. Wilcox, Factors affecting the ultrasonic intermodulation crack detection technique using bispectral analysis, NDT \& E International 41 (3) (2008) 223-234.

[25] W. Collis, P. White, J. Hammond, Higher-order spectra: the bispectrum and trispectrum, Mechanical systems and signal processing 12 (3) (1998) $375-394$.

[26] J. Jiao, J. Sun, N. Li, G. Song, B. Wu, C. He, Micro-crack detection using a collinear wave mixing technique, NDT \& E International 62 (2014) 122-129.

[27] C. Courtney, S. Neild, P. Wilcox, B. Drinkwater, Application of the bispectrum for detection of small nonlinearities excited sinusoidally, Journal of Sound and Vibration 329 (20) (2010) 4279-4293.

[28] N. Li, J. Sun, J. Jiao, B. Wu, C. He, Quantitative evaluation of microcracks using nonlinear ultrasonic modulation method, Ndt \& E International 79 (2016) 63-72.

[29] A. Sutin, P. Johnson, Nonlinear elastic wave nde ii. nonlinear wave modulation spectroscopy and nonlinear time reversed acoustics, in: AIP Conference Proceedings, Vol. 760, AIP, 2005, pp. 385-392.

[30] G. Zumpano, M. Meo, A new nonlinear elastic time reversal acoustic method for the identification and localisation of stress corrosion cracking in welded plate-like structures-a simulation study, International journal of solids and structures 44 (11-12) (2007) 3666-3684. 
[31] T. Goursolle, S. Dos Santos, O. B. Matar, S. Calle, Non-linear based time reversal acoustic applied to crack detection: Simulations and experiments, International Journal of Non-Linear Mechanics 43 (3) (2008) 170-177.

[32] A. Carcione, P. Blanloeuil, L. Rose, C. H. Wang, M. Veidt, Modulated high frequency excitation approach to nonlinear ultrasonic ndt, Journal of Sound and Vibration 446 (2019) 238-248.

[33] S. Ménigot, J.-M. Girault, I. Voicu, A. Novell, Optimization of contrastto-tissue ratio by frequency adaptation in pulse inversion imaging, IEEE transactions on ultrasonics, ferroelectrics, and frequency control 59 (11).

[34] S. Ménigot, J.-M. Girault, Optimization of contrast resolution by genetic algorithm in ultrasound tissue harmonic imaging, Ultrasonics 71 (2016) 231-244.

[35] S. Ménigot, M. Geryes, J. M. Girault, Inclusion/flaw detection in ultrasound imaging through optimization of random transmitted wave, in: Acoustics 2013, 2013.

[36] N. Houhat, S. Ménigot, T. Boutkedjirt, R. Drai, J.-M. Girault, Optimal stochastic excitation for linear flaw detection in a solid material, Lecture Notes in Computer Science 11401 (2019) 229-236.

[37] K. E. Van Den Abeele, A. Sutin, J. Carmeliet, P. A. Johnson, Microdamage diagnostics using nonlinear elastic wave spectroscopy (NEWS), Ndt \& E International 34 (4) (2001) 239-248. 
[38] V. Zaitsev, P. Sas, Nonlinear response of a weakly damaged metal sample: a dissipative modulation mechanism of vibro-acoustic interaction, Journal of Vibration and Control 6 (6) (2000) 803-822.

[39] B. Hilloulin, Y. Zhang, O. Abraham, A. Loukili, F. Grondin, O. Durand, V. Tournat, Small crack detection in cementitious materials using nonlinear coda wave modulation, NDT \& E International 68 (2014) 98-104.

[40] J.-B. Legland, Y. Zhang, O. Abraham, O. Durand, V. Tournat, Evaluation of crack status in a meter-size concrete structure using the ultrasonic nonlinear coda wave interferometry, The Journal of the Acoustical Society of America 142 (4) (2017) 2233-2241.

[41] A. H. Wright, Genetic algorithms for real parameter optimization, in: Foundations of genetic algorithms, Vol. 1, Elsevier, 1991, pp. 205-218.

[42] R. L. Haupt, S. E. Haupt, S. E. Haupt, Practical genetic algorithms, Vol. 2, Wiley New York, 1998.

[43] L. Costa, I. Santo, R. Denysiuk, E. Fernandes, Hybridization of a genetic algorithm with a pattern search augmented lagrangian method, 2010.

[44] J. Krautkrämer, H. Krautkrämer, Ultrasonic testing of materials, Springer Science \& Business Media, 2013.

[45] J. Riviere, G. Renaud, S. Haupert, M. Talmant, P. Laugier, P. A. Johnson, Probing interface elastic nonlinearity applying nonlinear resonance ultrasound spectroscopy: The case of screw tightness-of-fit, Journal of Applied Physics 107 (2010) 124901. 
[46] G. Chen, D. Pageot, J.-B. Legland, O. Abraham, M. Chekroun, V. Tournat, Numerical modeling of ultrasonic coda wave interferometry in a multiple scattering medium with a localized nonlinear defect, Wave Motion 72 (2017) 228-243.

[47] C. Payan, V. Garnier, J. Moysan, P. Johnson, Determination of third order elastic constants in a complex solid applying coda wave interferometry, Applied Physics Letters 94 (1) (2009) 011904.

[48] D. M. Egle, Diffuse wave fields in solid media, The Journal of the Acoustical Society of America 70 (2) (1981) 476-480.

[49] J. A. Ten Cate, T. J. Shankland, Slow dynamics in the nonlinear elastic response of berea sandstone, Geophysical Research Letters 23 (21) (1996) 3019-3022. 